\title{
A New Approach of Thin Goated Adsorbent Layer for Batch Adsorption Using Basic Dye
}

S.F. Azha ${ }^{1}$

A.L. Ahmad $^{1}$

S. Ismail ${ }^{*}, 1$

${ }^{1}$ School of Chemical Engineering, University Sains Malaysia, Engineering Campus, 14300 Nibong Tebal, Penang, Malaysia, Tel : +604-599 6458; Fax : +604-594 1013 *e-mail : chsuzy@usm.my

Clay based adsorbent which formulated to acts as thin coated adsorbent layer (TCAL) is a new approach for adsorption of coloured wastewater. In this study, TCAL has been evaluated for the removal of methylene blue dye as an adsorbate from aqueous solution. It was prepared by combination formula of commonly known clay based adsorbent, a binder as well as solvent. This paper focused on evaluation of TCAL in terms of performance including effects of different types of binder used and ratio of adsorbent to binder. The study effect on ratio was targeted for its coating strength, contact time as well as percentage dye removal. The experimental data reported that the water based binder was the most suitable binder to be used in TCAL in terms of adsorption performance and gave strength to the coating adsorbent itself. The optimum ratio of adsorbent to binder is $0.3 / 2.5$ weight mass. The percentage removal test for $100 \mathrm{mg} / \mathrm{L}$ concentration of Methylene Blue in batch adsorption of adsorbent coating layer was found to achieve $100 \%$ removal within less than 3 hours. Elemental analysis and morphological structure were also determined. The finding revealed the potential of TCAL as an effective adsorbent for undertaken adsorption process on basic dye.

Keywords : Clay, Binder, Thin coated adsorbent layer, Adsorbent coating, Basic dye, Adsorption.

\section{INTRODUCTION}

Research in coatings for various applications such as corrosion protection, aesthetics, wear resistance, thermal barrier, self-cleaning, antifouling and others have been very wide spread (Nwaogu \& Tiedje 2011), but there was still silent in the area of adsorbent coating for application on colour removal from aqueous solution in previous and recent years. It is a new approach and technique of treatment on textile wastewater and more specific on colour removal. This is because up to now, there is several treatment technologies have been developed and patented to 
decolorize dyeing wastewater. These treatment options include biological (Javaid et al. 2011), physio-chemical (Qian et al.2013), membrane filtration (Kurt et al. 2012), ozonation (Aziz et al. 2012) and advance oxidation (Yasar et al. 2013) and integrated treatment processes (Lotito et al. 2012). However, these processes face certain technical and economical limitations such as cost and production of sludge (Asgher \& Bhatti 2012).

Of these methods, adsorption has been found to be an efficient and economical process to remove dyes and also to control biological oxygen demand. Numerous studies have been devoted to sorption kinetics, equilibrium modeling, and mechanisms as well as to the factor that affect adsorption. Moreover, activated carbon is extensively used as an adsorbent due to its high degree of effectiveness (Wang et al. 2004). However, commercials activated carbon remained as an expensive materials and it also has high running costs since it required regeneration after adsorption). Hence there is a need for an equally effective but cheaper materials as an alternative adsorbent.

In recent years, clay has been accepted and emerged as one of the promising and appropriate low-cost adsorbents for colour wastewater treatment. Clays possess advantage properties that make them an ultimate choice as adsorbent for adsorption process such as low cost, high availability, and environmentally friendly material and subsequently can substitute expensive activated carbon and low performing bio-sorbent (Nassar et al. 2012). The adsorption proficiencies result from a net negative charge on the structure of clays. This negative charge give clay the capability to adsorb positively charged species. Their sorption properties also come from their high surface area and high porosity (Alkan et al. 2004). Nonetheless, the sorption capacity is higher for basic dye compare to acid dye due to character of the clay and ionic charges on the dyes (Crini 2006).

The advantages of adsorbent being coated on inert material or substrate have been mentioned long ago by Behan et al. (1992) since it is anticipated to have solid adsorbent deposited on a substrate as a coating instead of being confined in particulate form as pellets, beads or other particles. There are several reasons why solid adsorbent coating will be used, for example to improve the catalytic or adsorption properties of the solid adsorbent by improving the surface area to weight ratio, to reduce the amount of solid adsorbent required, to protect the underlying substrate material from a harmful environment, to achieve a particular strength or form and to perform the particular adsorptive or catalytic function over the entire coated surface of the substrate.

The study on coating had been carried out for fluoride removal in drinking water by Wu et al. (2008). The coating of Fe- Al$\mathrm{Ce}$ adsorbent with an acrylic-styrene copolymer latex as a binder to reinforce the adhesion strength and stability of the coating layer was developed and coated on sand by spraying. Sand is a promising selection as a carrier because of its high strength and low cost, which is $1 \%$ of the cost of the Fe-Al-Ce adsorbent. The 
granulated adsorbent obtained by spray coating Fe-Al-Ce adsorbent on the sand particle surface can be operated in the packed bed for drinking water treatment. This is because by comparing using Fe-AlCe in powder form which was only nanoscale in size, make the solid/liquid separation difficult, and the Fe-Al-Ce powder cannot be used in packed bed.

Beside, in another research study on coating, researchers also have developed techniques for coating an adsorbent onto sand to overcome the problem of using adsorbent powders in waste treatments process. Ion-oxide-coated sand (IOCS) has been tested for removing cations and anions from synthetic and natural waste. The results from their studies confirm that the utilization of iron-coated sand is worth developing for metal ions removal from water. This is because the usage of iron oxide alone is not suitable as a filter medium because of their low hydraulic conductivity and limited to reactor configurations incorporating with large sedimentation basins or filtration unit (Lai et al. 2000).

Several adsorption processes have been developed, patented and introduced for application in removing contaminants from waters (Crini 2006). Nevertheless, despite the number of published laboratory data and various low-cost adsorbents introduced by researchers, very few of these adsorption products and processes have been applied on industrial scale. There are several reasons in difficulty to transfer the process to industrial scale applications and one of the obvious reasons is the unwillingness of industries to invest on new equipment which upon their calculation require higher budget allocation continuously.

Therefore, this study was aimed at developing a new approach of adsorption through thin coated adsorbent layer for treatment of coloured wastewater. The development of clay-based adsorbent with binder will reinforce the adhesion strength and stability of the coating layer. This is the new approach in adsorption application by providing fundamental results at different angle of adsorption concept in colour removal technique.

\section{MATERIALS AND METHODS}

A synthetic textile dye, Methylene Blue (MB) supplied by Modern Lab Sdn. Bhd, Malaysia was an adsorbate and used as received without further purification. MB dye was made up in stock solution of concentration $1000 \mathrm{mg} / \mathrm{L}$ and was diluted to the required concentrations. The chemical formula of $\mathrm{MB}$ is $\mathrm{C}_{16} \mathrm{H}_{18} \mathrm{~N}_{3} \mathrm{SCI}$ with Colour Index 52015 and molecular weight of $319.85 \mathrm{~g} / \mathrm{mol}$.

\section{Preparation of adsorbent coating}

The sorbent used in this study was prepared by combination of clay-based adsorbent, binder and solvent with designated ratio. The mineral clay is laboratory grades purchased from Modern Lab Sdn. Bhd. Malaysia and also used as received without modification. The binder basically compose of calcium carbonate, polyvinyl alcohol, ammonia and hydrosol at different formulation: which are categorized under oil-based binder, latexbased binder and water-based binder. In general, the adsorbent coating was 
prepared by dissolving certain amount of adsorbent into measured amount of binder and solvent. The mixture was then magnetically stirred at ambient temperature for at least 15 minutes for complete homogeneous mixture.

\section{Effect on types of binder in TCAL}

In order to find out the best binder that can be used and mixed with clay-based adsorbent for the production of adsorbent coating, three types of binder have been used which were water-based, latex-based and oil-based binder. Adsorbent coating was prepared as stated in Section 2.2. The samples were then coated manually using paint brush around the interior part of 250 $\mathrm{ml}$ beaker. The beaker was then dried in an oven for 10 hours at $80^{\circ} \mathrm{C}$. The adsorption testing performance was carried out by filling the $50 \mathrm{mg} / \mathrm{L}$ concentration of $\mathrm{MB}$ into the coated beaker and stirring it using a highperformance multi-position magnetic stirrer with integrated temperature control plate (model RT 10 Power) at $130 \mathrm{rpm}$ and
$30{ }^{\circ} \mathrm{C}$ for certain contact time. The binder that showed the best adsorption performance was used for further experiment. The experimental apparatus for adsorbent coating is shown in Fig. 1.

\section{Effect of adsorbent to binder ratio for production of TCAL}

The best binder from Section 2.3.1 was chosen for further study. This study was conducted in order to find the best ratio for adsorbent coating from the result of coating strength of adsorbent layer, the performance of adsorption and its contact time. The designated ratio of adsorbent and binder was given in Table 1 . The amount of solvent was adjusted based on viscosity (material flow properties) of the solution mixture. The Viscometer DV-111 ULTRA Programmable Rheometer (Labtech) was used to measure the viscosity of the solution mixture. The best coating on surface cannot be too dilute and concentrate (range between 3 to $7 \mathrm{~g}$ of distilled water).
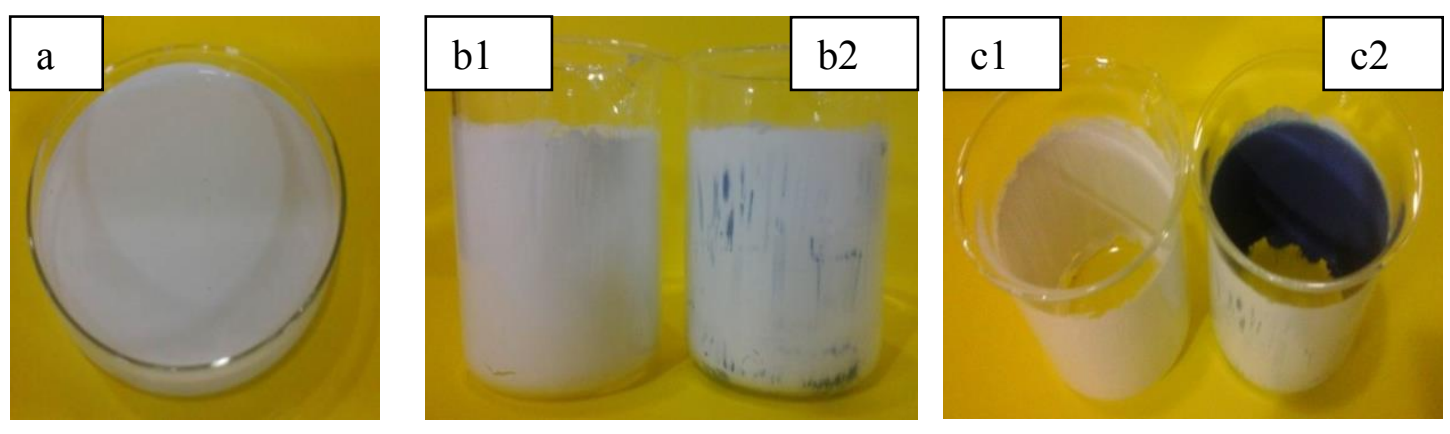

Fig. 1: (a) TCAL solution, (b1) front view of beaker being coated by TCAL, (b2) front view of beaker being coated by TCAL after adsorption process, (c1) upper view of beaker being coated by TCAL, (c2) upper view of beaker being coated by TCAL after adsorption process. 
Table 1. Composition of adsorbent and binder for adsorbent coating preparation. (study effect of composition ratio).

\begin{tabular}{cccccccccc}
\hline $\mathrm{A}(\mathrm{g})$ & $\mathrm{B}(\mathrm{g})$ & $\mathrm{A}(\mathrm{g})$ & $\mathrm{B}(\mathrm{g})$ & $\mathrm{A}(\mathrm{g})$ & $\mathrm{B}(\mathrm{g})$ & $\mathrm{A}(\mathrm{g})$ & $\mathrm{B}(\mathrm{g})$ & $\mathrm{A}(\mathrm{g})$ & $\mathrm{B}(\mathrm{g})$ \\
\hline 0.1 & & 0.1 & & 0.1 & & 0.1 & & 0.1 & \\
0.2 & & 0.2 & & 0.2 & & 0.2 & & 0.2 & \\
0.3 & 1.0 & 0.3 & 1.5 & 0.3 & & 0.3 & & 0.3 & \\
0.4 & & 0.4 & & 0.4 & 0.4 & & 0.4 & \\
0.5 & & 0.5 & & 0.5 & 0.5 & & 0.5 & \\
\hline
\end{tabular}

A(g): Adsorbent (g); B(g):Binder (g)

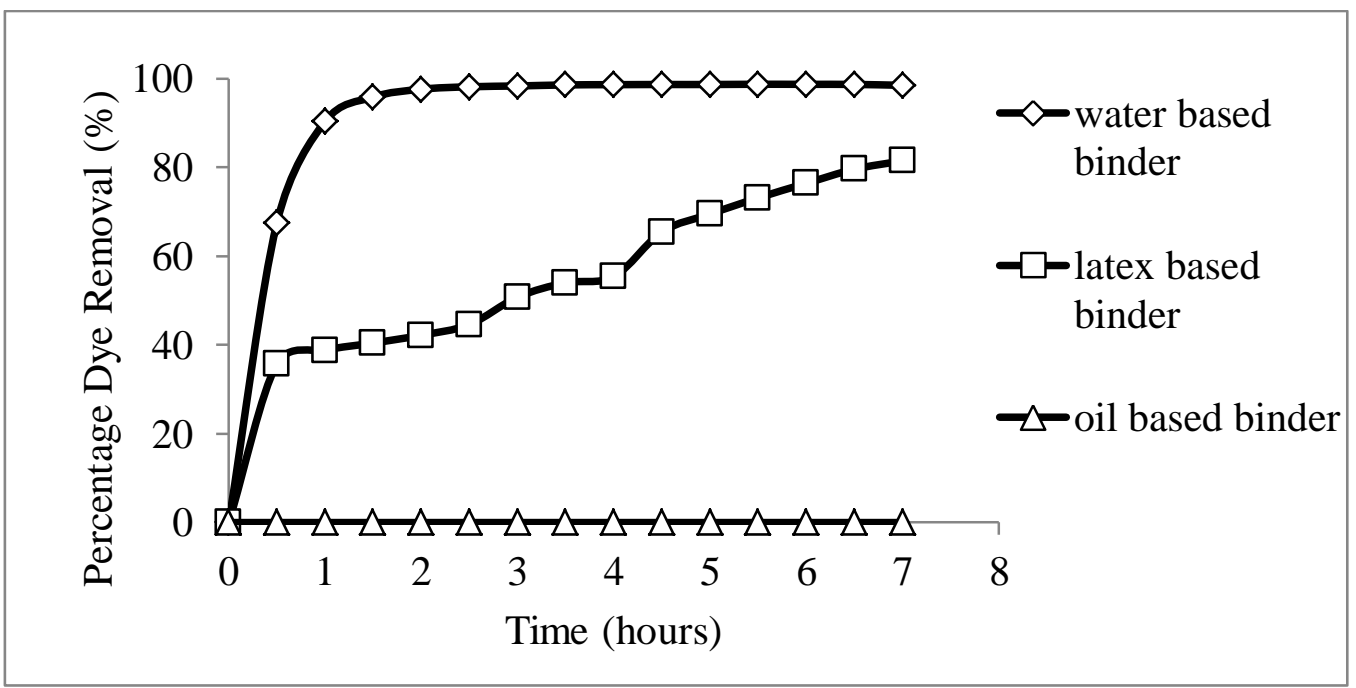

Fig. 2: Percentage dye removal of water-based, latex-based and oil-based binder.

\section{Characterization analysis}

The thin coated adsorbent layer was characterized for elemental analysis and surface morphology by scanning electron microscopy (SEM/EDX analyzer) (model Crest System (M) Sdn. Bhd., Quanta Feg 450) in order to study the dispersion of the clay-based adsorbent particles in the binder. The observation was carried out on top surface and cross-sectional surface of the samples.

\section{RESULT AND DISCUSSIONS Effect on types of binder in TCAL}

A simple screening study has been conducted to find out the best binder to be selected and mixed with adsorbent in order to evaluate the thin coated adsorbent layer. The three types (water based, latex based and oil based binder) of binders with similar ratio were coated on the surface and tested for its adsorption performance. The water and latex based binders gave better mixability with claybased adsorbent in the presence of distilled water as solvent. However, claybased adsorbent and water as solvent cannot be dissolved in oil based binder. Fig. 2 showed the adsorption performance for all types of binders. The adsorbent 
coating of water based binder gave the best and fastest adsorption compared to latex and oil-based binder. Within the first 2 hours, the adsorption reached $100 \%$ removal. Moreover, adsorbent coating with oil based binder unable adsorb the dye since the percentage dye removal is 0 $\%$ from 0 hour to the end of experiment. It might be due to the oil is as non-polar molecules while water molecules are polar in nature. Therefore, water molecules are positively charged. Since only opposites attract and oil has no charge at all, there is no force between the substances that allow them to mix. Latex based binder results showed a slow increment of adsorption until $80 \%$ removal was achieved within 7 hours of adsorption time. Hence, after having this simple test, the water based binder was chosen for the next study of physical characterization since it proved an excellent performance.

The morphology and behaviour of all types of binders were analysed using SEM images through cross sectional and surface area (Fig 3 (a)-(f)). Based on Fig 3 (a) and (b), it can be seen that water-based binder having a very loose structure, where it will ease the mixture of adsorbent once it being mixed together besides having pores. Therefore it is an advantage for this adsorbent coating since it will promote the formation of surface pores. However, the surface and cross sectional structure of latex-based binder (Fig.3 (c) and (d)) showed the compactness or dense of its structure and this will not allowed the adsorbent to be mixed with it, even the structure was much compact in oil-based binder (Fig. 3 (e) and (f)). The dense structure of binder will automatically clog the pore structure of clay-based adsorbent once it is being coated. This is due to the clay adsorbent having properties such as small particle size, high porosity, large surface area and high cation-exchange capacity.

\section{Effect of adsorbent/binder ratio}

Upon choosing the best binder for TCAL on removal of methylene blue, the effect of adsorbent to binder ratios were studied and was shown in Fig. 4 (a-e). The best ratio was based on the adsorption performance and coating strength, while the contact time was fixed up to 6 hours only. From the figures, the ratio between adsorbent and binder gave major effect towards adsorption performance as well as coating strength. It was observed that for the adsorbent/binder ratio of $0.1 / 2.5$, $0.2 / 2.5,0.3 / 2.5,0.4 / 2.4$ and $0.5 / 2.5$, the adsorption of $\mathrm{MB}$ towards adsorbent coating gave the best performance since all result achieving $100 \%$ removal. This can be simply attributed to the increased of sorbent surface area and availability of more adsorption site.

However, this result closely related to the coating strength. Even every ratio gave good performance, there were fluctuated and disrupted of adsorption performance result at every $0.5 \mathrm{~g}$ adsorbent amount. This had occurred due to the peel-off of coating layer from beaker's surface once $M B$ solution being introduced to the coated adsorbent. When the amount of adsorbent was higher, the amount of binder was assumed to be insufficient to bound adsorbent and make the adhesion of coated adsorbent with substrate or 

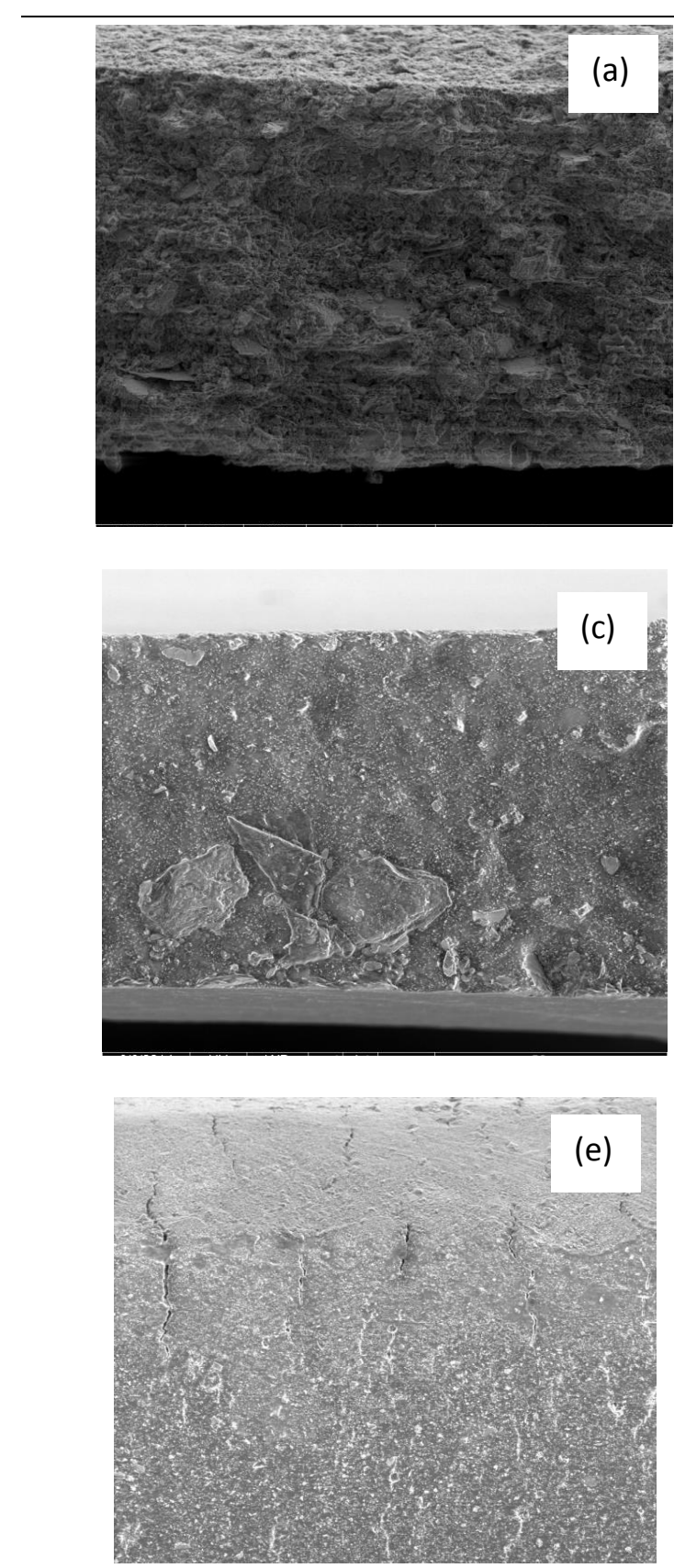
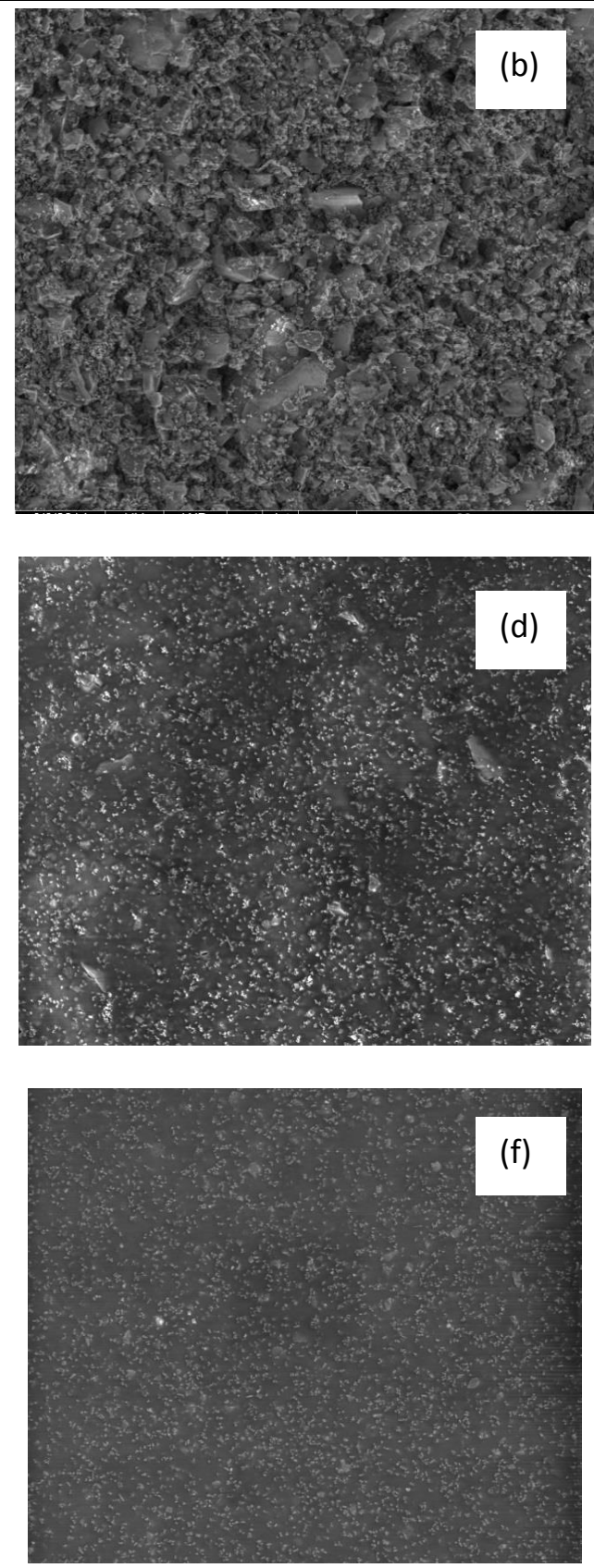

Fig. 3: Surface morphology of three types of binder (a) water-base binder (cross sectional), (b) water-base binder (top surface), (c) latex-base binder (cross sectional), (d) latex-base binder (top surface), (e) oil-base binder (cross sectional), (f) oil-base binder (top surface).

glass beaker decrease significantly. Subsequently, the stability of the adsorbent coating on surfaces also diminished in order the film to be formed. The binder plays a key role in increasing the adhesion among the adsorbent particles and between the particles and the glass surface.

Moreover, the percentage removal of $M B$ also decreased due to the blocking of the active site of adsorbent by binder. While looking at the adsorbent/binder 

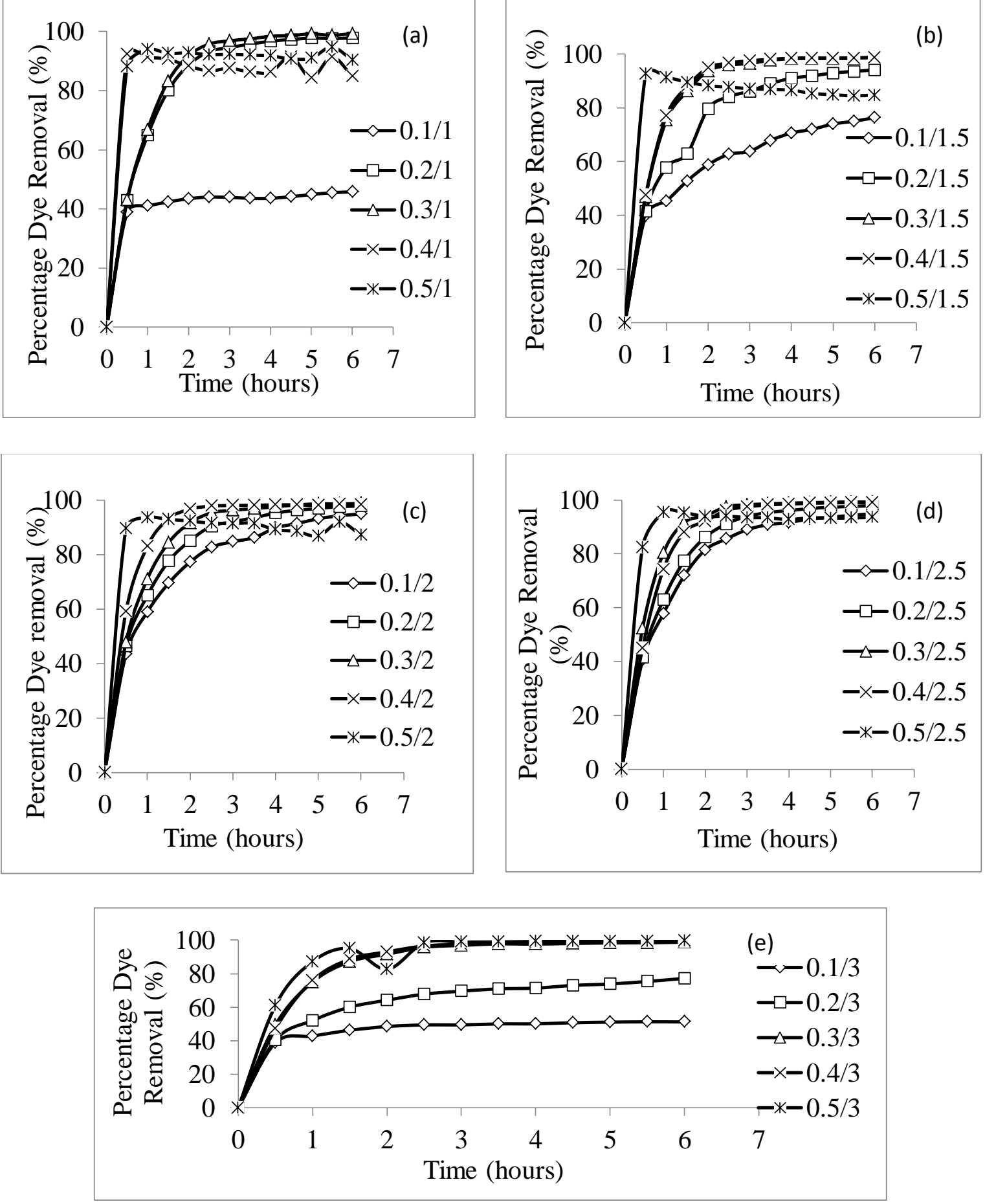

Fig. 4: The percentage dye removal (\%) of $M B$ corresponding to the ratio of adsorbent to binder. (a) adsorbent/binder $(0.1 / 1,0.2 / 1,0.3 / 1,0.4 / 1,0.5 / 1)$, (b) adsorbent/binder $(0.1 / 1.5,0.2 / 1.5,0.3 / 1.5,0.4 / 1.5,0.5 / 1.5)$, (c) adsorbent/binder $(0.1 / 2,0.2 / 2,0.3 / 2,0.4 / 2$, $0.5 / 2)$, (d) adsorbent/binder $\quad(0.1 / 2.5, \quad 0.2 / 2.5, \quad 0.3 / 2.5, \quad 0.4 / 2.5, \quad 0.5 / 2.5), \quad$ (e) adsorbent/binder $(0.1 / 3,0.2 / 3,0.3 / 3,0.4,3,0.5 / 3)$. 
Table 2. Result of the elements distribution image of EDX analysis.

\begin{tabular}{lcccccc}
\hline $\begin{array}{c}\text { Element determined } \\
\text { by EDX analysis }\end{array}$ & $\begin{array}{c}\text { Water- } \\
\text { based } \\
\text { binder }\end{array}$ & $\begin{array}{c}\text { Latex- } \\
\text { based } \\
\text { binder }\end{array}$ & $\begin{array}{c}\text { Oil-based } \\
\text { binder }\end{array}$ & $\begin{array}{c}\text { Clay- } \\
\text { based } \\
\text { adsorbent }\end{array}$ & TCAL & $\begin{array}{c}\text { TCAL } \\
\text { after } \\
\text { adsorption }\end{array}$ \\
\hline Carbon (C) & $/$ & $/$ & $/$ & $/$ & $/$ & $/$ \\
Oxygen (O) & $/$ & $/$ & $/$ & $/$ & $/$ & $/$ \\
Sodium (Na) & - & $/$ & - & $/$ & $/$ & - \\
Aluminium (Al) & $/$ & $/$ & $/$ & $/$ & $/$ & $/$ \\
Magnesium (Mg) & $/$ & - & - & $/$ & $/$ & $/$ \\
Silicon (Si) & $/$ & $/$ & $/$ & $/$ & $/$ & $/$ \\
Calcium (Ca) & $/$ & $/$ & $/$ & $/$ & $/$ & $/$ \\
Ferum (Fe) & - & - & - & $/$ & $/$ & $/$ \\
Titanium (Ti) & $/$ & $/$ & $/$ & - & $/$ & $/$ \\
Potassium (K) & $/$ & - & - & - & $/$ & $/$ \\
Sulphur (S) & - & - & - & - & - & $/$ \\
\hline
\end{tabular}

ratios of $0.1 / 1.0,0.1 / 1.5,0.1 / 2.0,0.1 / 2.5$ and $0.1 / 3.0$, the percentage dye removal was decreasing due to the lower number of adsorption site. It is important to determine the minimum quantity of the adsorbent, binder and solvent because too little may probably result in poor adhesion but, excess produces brittle coating which may crack on drying and peel-off during casting.

Besides that, contact time also play an important role in this adsorbent/binder ratio study. The selected best ratio also based on which ratio that showed the fastest adsorption process towards the dye pollutant. From the experiment, the contact time needed for $100 \mathrm{mg} / \mathrm{L} \mathrm{MB}$ solution to reach equilibrium was less than 3 hours for some ratios. The result of the adsorption was considered fast. Therefore, by taking into consideration of the effect of adsorption performance, coating strength and contact time, the adsorbent/binder ratio was set at 0.3/2.5 as the optimum value for the subsequent experiment. The viscosity for the optimum ratio was 190.07 centipoise.

The energy dispersive X-ray (EDX) analysis was also carried out on 6 samples which were (a) water-based binder, (b) latex-based binder, (c) oil-based binder, (d) clay-based adsorbent, (e) TCAL and (f) TCAL after adsorption using the scanning electron microscope and was presented in Fig. 5 . As seen in Fig. 5 and Table 2, the detected elements in all samples are Carbon (C), Oxygen (O), Aluminium (Al), Calcium (Ca), and Silicon ( $\mathrm{Si}$ ). From the literature, $\mathrm{SiO}_{2}$ and $\mathrm{Al}_{2} \mathrm{O}_{3}$ are the major constituents of the clay with other oxides present in trace amounts, so it is expected either any of the major oxides present in the adsorbent or their combined influence are responsible for the adsorption of dye. Even the major elements also contained in all types of binders, it still gave different effect towards the adsorption performance. This is due to the difference in amount of the elements as well as the presence of other component that 

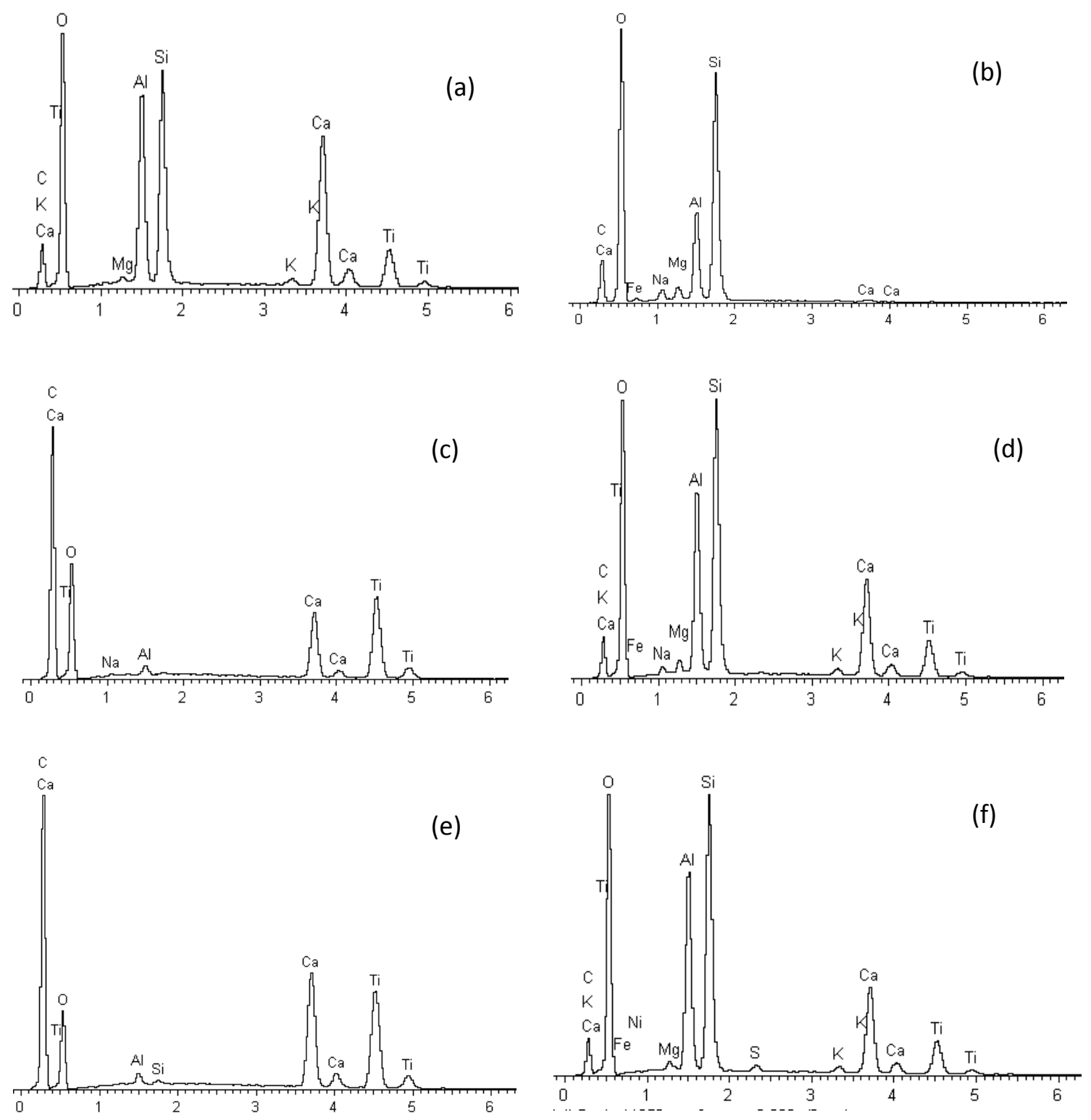

Fig. 5: EDX graph for every major element in (a) Water-based binder, (b) Latex-based binder, (c) Oil-based binder, (d) Clay-based adsorbent, (e) TCAL, (f) TCAL after adsorption.

clogged the pore structure of adsorbent, e.g, oil-based binder is made from vegetables oil that dries or crosslinks when it is exposed to the air and then form a film or coated layer. In Fig. 5(f), the element of sulphur was found exist and this was obtained from MB element as an adsorbent in this study. The chemical formula for $\mathrm{MB}$ is $\mathrm{C}_{16} \mathrm{H}_{18} \mathrm{~N}_{3} \mathrm{SCl}$.

\section{CONCLUSION}

This study presented the tendency of TCAL as an effective adsorbe (b) ating to be used in adsorption technique for colour removal. There are several reasons why study has stress on the thin coated layer of adsorbent instead of being contained in particulate form as pellets, beads, powder 
or other particles. At first, the thin coated adsorbent layer can perform the particular adsorptive or catalytic function over the entire coated surface of the substrate. Therefore, this can improve the catalytic or adsorption properties of the solid adsorbent by improving the surface area to weight ratio. Since TCAL applied the thin film concept, thus it leads to the increasing wide aperture surface area of this adsorbent. Besides that, it also gives advantages in reducing the amount of solid adsorbent required. By having a very simple mixture of adsorbent, binder and solvent, the usage of clay-based adsorbent can be reduce and indirectly produce a low cost TCAL. In conclusion, thin coated adsorbent layer opens to a new paradigm in adsorption application that will give option to industries meeting their requirement. The adsorption of $\mathrm{MB}$ by TCAL was found to be very effective and innovative for further coloured wastewater treatment technology. Further study on adsorption using TCAL of various types of dye or actual textile wastewater will be taking into consideration.

\section{ACKNOWLEDGEMENTS}

The authors would like to acknowledge the Fundamental Research Grant Scheme, FRGS (203/PJKIMIA/6071253), Membrane for Science and Technology (MST) Cluster and Kementerian Pengajian Tinggi for providing MyMaster for the financial support.

\section{REFERENCES}

1. Nwaogu, U. C., \& Tiedje, N. S. (2011). Foundry Coating Technology: A Review.Materials Sciences \& Applications, 2(8).

2. Javaid, M., Saleemi, A. R., Naveed, S., Zafar, M., \& Ramzan, N. (2011). Anaerobic treatment of desizing effluent in a mesophilic anaerobic packed bed reactor. Journal of the Pakistan Institute of Chemical Engineers, 39(1), 61-67.

3. Qian, F., Sun, X., \& Liu, Y. (2013). Removal characteristics of organics in bio-treated textile wastewater reclamation by a stepwise coagulation and intermediate $\mathrm{GAC} / \mathrm{O}<$ sub $>$ $3</$ sub $>$ oxidation process. Chemical Engineering Journal, 214, 112-118.

4. Kurt, E., Koseoglu-Imer, D. Y., Dizge, N., Chellam, S., \& Koyuncu, I. (2012). Pilotscale evaluation of nanofiltration and reverse osmosis for process reuse of segregated textile dyewash wastewater. Desalination, 302, 24-32.

5. Aziz, F., Rehman, M. S. U., Batool, A., Muhammad, A., \& Mahmood, T. (2012). Pretreatment of municipal, industrial and composite wastewater by ozonation. Environ. Process. Eng, 1, 2.

6. Yasar, A., Khalil, S., Tabinda, A. B., \& Malik, A. (2013). Comparison of cost and treatment efficiency of solar assisted advance oxidation processes for textile dye bath effluent. Korean Journal of Chemical Engineering, 30(1), 131-138. 
7. Lotito, A. M., Fratino, U., Bergna, G., \& Di Iaconi, C. (2012). Integrated biological and ozone treatment of printing textile wastewater. Chemical Engineering Journal, 195, 261-269.

8. Asgher, M., \& Bhatti, H. N. (2012). Evaluation of thermodynamics and effect of chemical treatments on sorption potential of Citrus waste biomass for removal of anionic dyes from aqueous solutions. Ecological Engineering, 38(1), 79-85.

9. Wang, C. C., Juang, L. C., Hsu, T. C., Lee, C. K., Lee, J. F., \& Huang, F. C. (2004). Adsorption of basic dyes onto montmorillonite. Journal of colloid and interface science, 273(1), 80-86.

10. Nassar, M. M., El-Geundi, M. S., \& AlWahbi, A. A. (2012). Equilibrium modeling and thermodynamic parameters for adsorption of cationic dyes onto Yemen natural clay. Desalination and Water Treatment, 44(1-3), 340-349.

11. Alkan, M., Demirbaş, Ö., Celikcapa, S., \& Doğan, M. (2004). Sorption of acid red 57 from aqueous solution onto sepiolite. Journal of hazardous materials,116(1), 135-145.
12. Crini, G. (2006). Non-conventional lowcost adsorbents for dye removal: a review. Bioresource technology, 97(9), 1061-1085.

13. Behan, A. S., Cohen, A. P., Dunne, S. R., \& McKeon, M. J. (1992). U.S. Patent No. 5,120,694. Washington, DC: U.S. Patent and Trademark Office.

14. Wu, H. X., Wang, T. J., Dou, X. M., Zhao, B., Chen, L., \& Jin, Y. (2008). Spray coating of adsorbent with polymer latex on sand particles for fluoride removal in drinking water. Industrial \& Engineering Chemistry Research, 47(14), 4697-4702.

15. Lai, C. H., Lo, S. L., \& Chiang, H. L. (2000). Adsorption/desorption properties of copper ions on the surface of iron-coated sand using BET and EDAX analyses.Chemosphere, 41(8), 12491255. 